\title{
Mission Reliability Estimation for Repairable Robot Teams
}

\author{
Stephen B. Stancliff ${ }^{1}$, John M. Dolan ${ }^{1}$, and Ashitey Trebi-Ollennu ${ }^{2}$ \\ ${ }^{1}$ The Robotics Institute, Carnegie Mellon University, Pittsburgh, PA, USA \\ stancliff@cmu.edu, jmd@cs.cmu.edu \\ 2 Jet Propulsion Laboratory, Pasadena, CA, USA \\ Ashitey.Trebi-Ollennu@jpl.nasa.gov
}

\begin{abstract}
NASA has expressed interest in using modular self-repairable robotic teams for the exploration and colonization of Mars. One of the reasons often given for using repairable robots is increased reliability. Analytical tools are needed for estimating the reliability of robotic missions in order to determine if this reasoning is correct, and for what types of missions. In this paper we present the first method for analytically predicting the probability of mission completion for teams of repairable mobile robots. We then apply this method to compare the reliability of repairable and nonrepairable robot teams for an example mission scenario. Our results show that for this simple mission, with reasonable assumptions regarding costs, teams of repairable robots with spare components are superior to teams with spare nonrepairable robots.
\end{abstract}

\section{Introduction}

The NASA Exploration Systems, Human \& Robotic Technology (H\&RT) Formulation Plan identifies Strategic Technical Challenges which "must be surmounted to enable sustainable future human and robotic exploration" of our solar system [1]. These include robotic networks, modularity, reconfigurability, reusability, and redundancy. The plan further identifies the need for Intelligent Modular Systems enabling safe, affordable, effective, multifunctional robotic technologies for sustainable human and robotic exploration to meet the U.S. National Vision for Space Exploration.

Modularity, reconfigurability, reusability, and redundancy add new complexity to the mission design process for robotic exploration. Decisions must be made about how to divide tasks among robots, how many robots to use, and how to configure individual robots in order to accomplish individual tasks and overall mission goals.

A significant factor in making these decisions is the impact of robot failures on mission completion. The literature (e.g., [2]) indicates that field robots have poor reliability, with robots being unavailable approximately half of the time. In contrast, the planetary rovers built by NASA have very high reliability, but this reliability is achieved at very high cost. Sending teams of robots to Mars while keeping costs down will require the design of robots with enough reliability to accomplish the mission but without excess reliability. 
The general problem that we would like to solve is: "What is the lowest-cost configuration of robots that will accomplish a given set of mission tasks with a given probability of success?" In considering robot team configurations we wish to compare repairable versus nonrepairable robots, different component reliabilities, different repair strategies, different numbers of robots, and different numbers of spare parts.

The only known previous work studying how cooperative repair impacts the reliability of robot team missions is [3]. That paper's methods are similar to ours in being based in the reliability literature, but significantly different in assuming that repair incurs no cost in terms of time and reliability. We contend that in most cases this cost of repair is significant - the robots executing the repair must delay their assigned task in order to perform a repair, and the act of repair increases their own chance of failure.

Additionally, [3] considers only cannibalistic repair, where all replacement parts are scavenged from failed robots, and all spares are carried by the surviving robots. Our method has been designed to be flexible with respect to the type of repair.

Finally, [3] leaves open the question of whether repairability is cost-effective. If a repairable team can do $25 \%$ more work but increases the mission cost by $75 \%$, then it may not be the superior option. We incorporate cost into our evaluation method, qualitatively in this paper, and quantitatively in future work.

In [4] we present a method for quantifying the reliability of robot modules and individual robots. In this paper, we begin to address how these reliability tools can be used to evaluate mission design alternatives for robot teams. In Sections 2 and 3, we outline a simple mission scenario and our method of representing it. In Section 4, we derive analytical solutions for the probability of mission success for this mission using repairable and nonrepairable robot teams. In Section 5, we apply our methodology to compare different alternatives for improving the reliability of an example mission.

\section{Problem Representation}

We treat both repairable robots (RR) and nonrepairable robots (NR) as being constructed of multiple hardware modules. A robot might, for instance, be composed of a computation module, a propulsion module, and a manipulation module. A robot fails when one of its constituent modules fails. For NR, failure is terminal. For RR, the failed module can be replaced by a spare module if one is available. The module replacement procedure is carried out by a robot other than the failed robot.

The probability of a module's failing is found using standard reliability engineering methods assuming a constant hazard rate. Two inputs determine the module failure probability: the module's failure rate, often given by mean time to failure (MTTF), and the length of time the module is operated. Ref. [4] gives more details on the calculation of module and robot failure.

We have begun our analysis of robot mission reliability by examining a seemingly simple mission - a group of robots must traverse together for some days, and all of them must be functioning at the end of the traverse. We specify variants of this mission using the nomenclature $(N, D, M)$, where $N$ is the number of robots, $D$ the number of days, and $M$ the number of spare hardware modules available. A mission with two robots traversing for one day with no spares available is described as mission $(2,1,0)$. 
The space of all paths that could be followed by the robots can be represented in tree form. Fig. 1 (left) shows the tree for mission $(2,1,0)$. After a task node (Transit in this case), the state of the robots is evaluated. Since there are no spare modules available for $(2,1,0)$, the mission results in failure if either robot fails.

With spare modules, not all robot failures result in mission failure, so we must examine more alternatives at each node. With two RR (Fig. 1, center), the possible outcomes for each node are (a) both robots are alive, (b) robot 1 is alive and 2 is dead, (c) robot 1 is dead and 2 is alive, and (d) both robots are dead. Outcome (a) causes the robots to continue with the original (nonrepairable) plan. For (d) the mission fails because there are no functioning robots available to perform a repair. For (b) and (c) the robots must execute a repair sequence before returning to the original plan.

In Fig. 1 (right) we abstract further from the repair details by considering the status of the team, rather than individual robots. The symbol "+" means that all robots are alive, and "-" means that one or more, but not all, of the robots have failed.

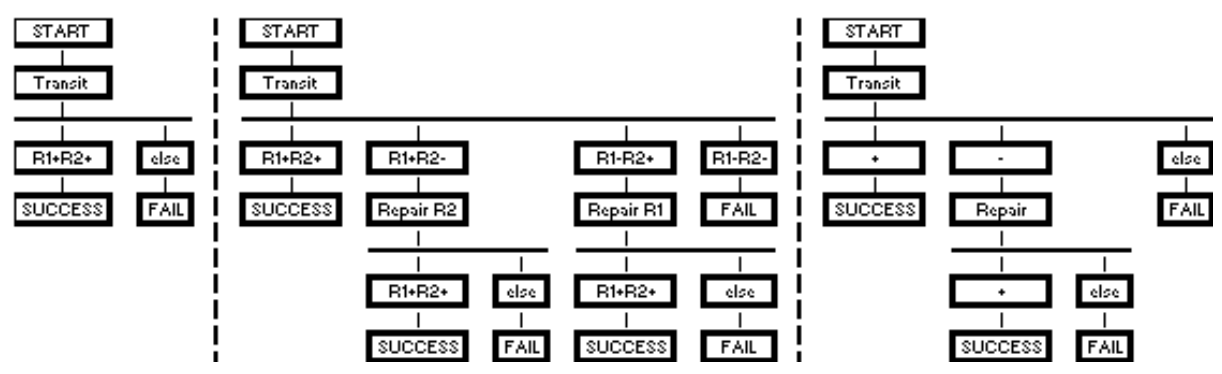

Fig. 1. (left) Mission (2,1,0); (center) Mission (2,1,1); (right) Mission (N,1,1)

We can use these trees to calculate the probability of mission success (PoMS) for the represented team configurations. The probability of reaching a leaf node is calculated by traversing from the root node to that leaf and multiplying the probabilities for the nodes traversed. In Fig. 1 (center), the probability of reaching the second success node is the probability that robot 1 is alive and robot 2 is dead after the Transit action multiplied by the probability that both robots are alive after the Repair $R 2$ action. The PoMS is the sum of the probabilities for the three success nodes.

\section{Simplifications}

In our representation robot failure is assumed to occur at the end of a task. This allows us to avoid dealing with partially completed tasks. This discretization does not limit the resolution of the representation, because the tasks can be restated into subtasks if smaller time increments are needed.

In repairing robots we ignore the different types of spare modules. The spares are considered as a store of universal replacements. If five spares are available, we can replace five of module $\mathrm{A}$, or three of module $\mathrm{A}$ and two of module $\mathrm{B}$, or any other combination. We do consider module differences when calculating failures; i.e., the different modules making up a robot contribute differently to the probability of the 
robot failing. This is a significant simplification which favors the repairable teams. We revisit this simplification in the example and intend to eliminate it in future work.

We treat all repair activities as having the same cost, in terms of failure rates. In reality, the amount of work required to replace Module A may be different from that for Module B. Further, under many repair scenarios the cost of repair may be a function of location, e.g., if the spare modules are kept at depots. This simplification is reasonable if the robots carry the spares with them (which is feasible only for small numbers of spares) and if different modules require equal effort for replacement.

We ignore simultaneous failure of modules or robots, other than complete failure of the robot team. After each task, we ask, "did one or more (but not all) robots fail?" If the answer is yes, then we perform a single repair, using a single replacement module. If in fact more than one robot was calculated to fail after a task, then the additional robots are assumed to be repaired instantly and without use of resources. This assumption is reasonable for small teams and short missions if the robots and modules are inherently very reliable since, in that case, the probability of simultaneous failure of modules or robots is very small.

\section{Analytical Solutions}

For the mission described above we are able to derive a general expression for the PoMS for an RR team in terms of $N, D$ and $M$. Consider first an NR team as in Fig. 1 (left). The probability of reaching the success node is simply the probability that all robots are alive after the transit task, i.e., $\mathrm{P}(N, 1,0)=T^{+}$.

Moving to the configuration of Fig. 1 (right), the probability of reaching the first success node is $T^{+}$. The probability of reaching the second success node is the probability that a robot is dead (but not all robots are dead) after the transit task, multiplied by the probability that the ensuing repair task succeeds, or $T^{-} R^{+}$. The overall PoMS is $P(N, 1,1)=T^{+}+T^{-} R^{+}$. Similarly we find that $P(N, 2,1)=\left(T^{+}\right)^{2}+2\left(T^{+}\right)\left(T^{-} R^{+}\right)$and $P(N, 3,1)=\left(T^{+}\right)^{3}+3\left(T^{+}\right)^{2}\left(T^{-} R^{+}\right)$. We can generalize as

$$
P(N, D, 1)=\left(T^{+}\right)^{D}+D\left(T^{+}\right)^{(D-1)}\left(T^{-} R^{+}\right) .
$$

Proceeding in the same way for teams with two spare modules gives

$$
\begin{gathered}
P(N, 1,2)=\left(T^{+}\right)+\left(T^{-} R^{+}\right)+\left(T^{-} R^{+}\right) R^{-}, \\
P(N, 2,2)=\left(T^{+}\right)^{2}+2\left(T^{+}\right)\left(T^{-} R^{+}\right)+2\left(T^{+}\right)\left(T^{-} R^{+}\right) R^{-}+\left(T^{-} R^{+}\right)^{2}, \\
P(N, 3,2)=\left(T^{+}\right)^{3}+3\left(T^{+}\right)^{2}\left(T^{-} R^{+}\right)+3\left(T^{+}\right)^{2}\left(T^{-} R^{+}\right) R^{-}+3\left(T^{+}\right)\left(T^{-} R^{+}\right)^{2}, \\
P(N, 4,2)=\left(T^{+}\right)^{4}+4\left(T^{+}\right)^{3}\left(T^{-} R^{+}\right)+4\left(T^{+}\right)^{3}\left(T^{-} R^{+}\right) R^{-}+6\left(T^{+}\right)^{2}\left(T^{-} R^{+}\right)^{2}, \text { and }
\end{gathered}
$$




$$
P(N, D, 2)=\left(T^{+}\right)^{D}+D\left(T^{+}\right)^{D-1}\left(T^{-} R^{+}\right)+D\left(T^{+}\right)^{D-1}\left(T^{-} R^{+}\right) R^{-}+{ }^{D} C_{2}\left(T^{+}\right)^{D-2}\left(T^{-} R^{+}\right)^{2},
$$

where ${ }^{D} C_{2}$ is the number of combinations of 2 in D.

Similarly we find that

$$
P(N, D, 3)=\left(T^{+}\right)^{D}+\left\{\begin{array}{l}
D\left(T^{+}\right)^{D-1}\left(T^{-} R^{+}\left[1+R^{-}+R^{-2}\right]\right. \\
+{ }^{D} C_{2}\left(T^{+}\right)^{D-2}\left(T^{-} R^{+}\right)^{2}\left[1+R^{-}\right]+{ }^{D} C_{3}\left(T^{+}\right)^{D-3}\left(T^{-} R^{+}\right)^{3}
\end{array}\right\} .
$$

Examining eq. (1), (2) and (3) we determine that

$$
P(N, D, M)=\left(T^{+}\right)^{D}+\sum_{j=1}^{M}\left[{ }^{D} C_{j}\left(T^{+}\right)^{D-j}\left(T^{-} R^{+}\right)^{M-j} \sum_{i=0}^{M}\left(R^{-}\right)^{i}\right] .
$$

We also consider teams of NR where there are spare robots. This allows us to compare the cost of building repairable robots and sending spares versus sending larger numbers of $\mathrm{NR}$. We represent this team configuration as $(n \mid N, D, 0)$, where $n$ is the number of robots required to survive. For the configuration $(2 \mid 3, D, 0)$ we need at least two robots out of three to survive after $D$ days. The probability of all three robots surviving is $P(3, D, 0)=\left(T_{3}^{+}\right)^{D}$, where $T_{3}^{+}$represents the probability that a team with three members has all robots alive after the transit task. The probability that exactly two robots survive for $D$ days is $\left(T_{2}^{+}\right)^{D}\left[1-\left(T_{1}^{+}\right)^{D}\right]$. The overall PoMS is therefore

$$
P(2 \mid 3, D, 0)=\left(T_{3}^{+}\right)^{D}+3\left(T_{2}^{+}\right)^{D}\left[1-\left(T_{1}^{+}\right)^{D}\right] .
$$

Similarly, for four robots we get

$$
P(2 \mid 4, D, 0)=\left(T_{4}^{+}\right)^{D}+4\left(T_{3}^{+}\right)^{D}\left[1-\left(T_{1}^{+}\right)^{D}\right]+6\left(T_{2}^{+}\right)^{D}\left[1-\left(T_{2}^{+}\right)^{D}\right] .
$$

\section{Example Application}

We now examine a simple mission design problem comparing the reliability of RR and NR teams. We describe the mission to be analyzed, then calculate the PoMS for alternative robot team configurations, and finally compare the cost of the alternatives.

The mission requires two robots to be alive after a six-day traverse, with PoMS $=0.99999$. All robots are identical and are composed of three modules: (A) propulsion, (B) computation/power and (C) manipulation. The MTTF for the modules are $50000 \mathrm{~h}, 40000 \mathrm{~h}$, and $30000 \mathrm{~h}$, respectively, and the numbers of hours each module is used for the Transit task are 6,6 and 0, respectively.

The simplest available team configuration uses two NR. The probabilities of survival for each module after the Traverse task are calculated with the MTTF and usage given above and are found to be $\mathrm{P}(\mathrm{S})_{\mathrm{A}}=0.99988, \mathrm{P}(\mathrm{S})_{\mathrm{B}}=0.99985$ and $\mathrm{P}(\mathrm{S})_{\mathrm{C}}=1$. The probability of survival of an entire robot after the traverse action is 
$\mathrm{P}\left(\mathrm{T}_{1}^{+}\right)=\mathrm{P}(\mathrm{S})_{\mathrm{A}} \cdot \mathrm{P}(\mathrm{S})_{\mathrm{B}} \cdot \mathrm{P}(\mathrm{S})_{\mathrm{C}}=0.99973$. The probability of survival of two robots after six traverses is $\mathrm{P}(\mathrm{S})=\left(\left(\mathrm{P}\left(\mathrm{T}_{1}^{+}\right)^{2}\right)^{6}=0.99676\right.$. Therefore, this configuration falls short of the mission reliability requirement.

One option for improving the PoMS is to use more reliable modules. We could meet the PoMS requirement of 0.99999 by increasing the MTTF of modules A and B to $13,800,000$ hours.

Another option is to use more than two robots, with only two robots needing to be alive at the end of the six days. Using eq. (5) for a team of three robots yields $\mathrm{P}(\mathrm{S})=\mathrm{P}(2 \mid 3,6,0)=0.999992$.

The final option we examine is to use RR and spare modules. We assume here that the spares are carried on the robots, and ignore the negative effects of carrying those modules on the reliability of the Transit task. During the Repair task the module usages for the robot being repaired are $A=3, B=1, C=1$ (all in hrs), and the usages for the robot performing the repair are $A=3, B=3, C=2$. Evaluating eq. (4) for two robots and one spare module we find that $\mathrm{P}(\mathrm{S})=0.999994$.

The PoMS for each configuration are shown in Table 1. The meaning of small differences here is not intuitive, so we also provide another way of looking at them which is "How many days can each configuration traverse with the required $\mathrm{P}(\mathrm{S})=0.99999$ ?" We see here that the base configuration fails to meet the required mission duration and reliability, options $a$ and $b$ just meet the mission requirements, and option $c$ provides two extra days of operation above the mission requirements.

Table 1. PoMS and days of operation for each option

\begin{tabular}{|c|c|c|c|c|}
\hline & Base & (a) Increase MTTF & (b) 3NR & (c) 2RR+1M \\
\hline $\mathrm{P}(\mathrm{S})$ & 0.99676 & 0.99999 & 0.999992 & 0.999994 \\
\hline Days & 0 & 6 & 6 & 8 \\
\hline
\end{tabular}

In a real-world mission design scenario we would be able to compare the expected cost of the three options in order to determine the lowest cost alternative. Lacking real cost information we will instead make qualitative arguments about the relative costs of the alternatives.

Option $a$ requires the reliability of the component modules to be increased by three orders of magnitude. Such an increase in component reliability is unlikely, especially for a system that has already been designed to the standards required for planetary exploration. Even if possible, the cost of such an improvement would be very high.

Option $b$ requires the construction and deployment of an additional robot. The construction cost for one additional robot should be relatively small, perhaps $25 \%$ of the cost of developing the first robot. However, the cost to transport an additional robot to Mars is very large, on the order of $\$ 100 \mathrm{M}$.

Option $c$ requires the addition of self-repair capabilities to the robot. We assume here that the requisite technologies are available, so the cost is simply that of implementing these technologies on the specific robot platform. We estimate that this cost will add $25 \%$ to development costs. Option $c$ also requires the transport of one additional robot module. This cost is significant but is much less than the cost of transporting an entire robot.

We therefore conclude that option $a$ is infeasible, and option $c$ appears to be preferable to option $b$ when transportation costs are high. In other applications where 
transportation costs are low, the costs of options $b$ and $c$ may be comparable, in which case other factors would determine the choice. One factor would be the lower technological risk of option $b$.

A caveat here is the "universal module" simplification described earlier. This allows us to replace a failed module of any type with a single spare. In reality, in order to assure that we can replace a single failed module, we need one replacement module of each type. In that case the transportation savings of option $c$ over option $b$ will diminish significantly. However, these additional modules will also increase the PoMS of the repairable team. This would allow us to use modules with lower reliability and lower cost. The conclusion therefore remains the same-spare modules are preferable to spare robots. Further work is needed to remove this simplification.

Fig. 2 compares different team configurations for missions of varying length. We see that teams with spare NR and teams with RR plus spare modules are all far superior to the team of two NR. We also see that two RR with one spare module is slightly better than three NR, and that two RR with two spare modules is almost as good as four NR.

Finally, Fig. 3 shows the same team configurations and repair reliabilities as Fig. 2 , but with the MTTF of all components reduced by half. The performance of all teams is reduced, but the RR teams are reduced less than the NR teams. This shows that the advantage of RR teams is greater when the underlying mission (i.e., the modules and their usage) has lower reliability.

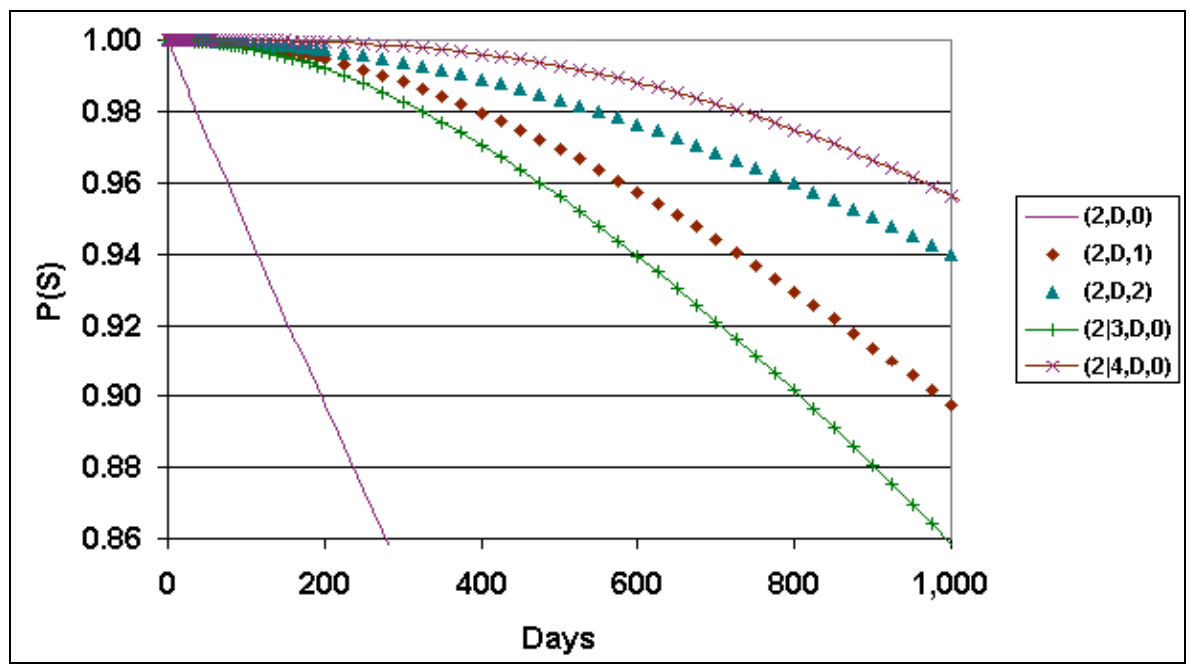

Fig. 2. Mission success for different team configurations

\section{Summary and Future Work}

In this paper we present the first method for analytically predicting the PoMS for teams of repairable and nonrepairable mobile robots. With further development, this method and its supporting tools should allow a mission designer to make informed 
comparisons between team configurations during the early stages of mission design.

We believe that the methods used in this paper can be applied to a variety of robot mission design problems. However, the amount of work required to derive an analytical solution for even a simple mission is significant, and generating the trees themselves is a significant task for nontrivial missions. A focus of future work is to investigate methods for solving complex missions in an automated way.

In future work we also must address the simplifications listed in Section 3. In particular, we must differentiate the store of modules and deal with simultaneous failures so that we can fully evaluate how many modules of each type are required to achieve a certain PoMS.

Finally, we would like to find or develop a model of robot cost. Our goal is to be able to make design decisions based on tradeoffs between cost and reliability. In order to make convincing arguments about the superiority of one team configuration over another, we need better cost estimates than the rough approximations that we used in the example in this paper.

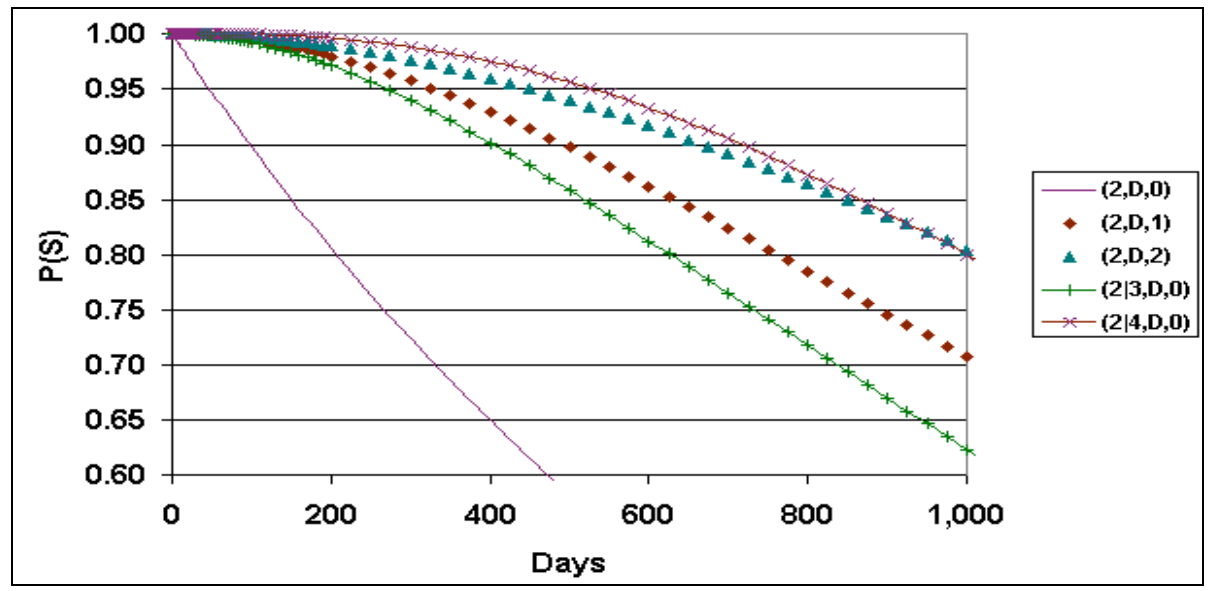

Fig. 3. Mission success for team configurations with decreased transit reliability

\section{References}

1. NASA (National Aeronautics and Space Administration), 2004. Human \& Robotic Technology (H\&RT) Project Formulation Plan, Version 5.1. Retrieved June 25, 2005 from NASA Exploration web site: http://exploration.nasa.gov/documents/nova_29july04b.pdf

2. Carlson, J., and Murphy, R., 2003. Reliability Analysis of Mobile Robots. In: Proc. 2003 IEEE Int'l Conf. Robotics and Automation (ICRA 2003), September 14-19, 2003, Taipei, Taiwan. IEEE, 274-281.

3. Bererton, C., and Khosla, P., 2002. An Analysis of Cooperative Repair Capabilities in a Team of Robots. In: Proc. 2002 IEEE Int'l Conf. Robotics and Automation (ICRA 2002), May 11-15, 2002, Washington, DC, USA. IEEE. 476-486.

4. Stancliff, S.B, Dolan, J.M., and Trebi-Ollennu, A., 2005. Towards a Predictive Model of Mobile Robot Reliability. To be published as tech. report, The Robotics Institute, Carnegie Mellon University, 2005. 